\title{
Arquitectura para el análisis de estados financieros XBRL publicados por empresas en México utilizando lógica difusa
}

\author{
Cristian Noé Enríquez-Marcial ${ }^{1}$, Hilarion Miñoz-Contreras ${ }^{1}$, \\ José Luis Sánchez-Cervantes ${ }^{2}$, Lisbeth Rodríguez-Mazahua ${ }^{1}$, Giner Alor-Hernández ${ }^{1}$ \\ ${ }^{1}$ División de estudios de Posgrado en Invertigación, \\ Instituto Tecnológico de Orizaba, \\ México \\ ${ }^{2}$ CONACYT - Instituto Tecnológico de Orizaba, \\ México \\ \{marcial2005, lisbethr08\}@gmail.com, hmunoz189@msn.com, gineralor@outlook.com, \\ jsanchez@ito-depi.edu.mx
}

\begin{abstract}
Resúmen. El análisis de estados financieros es el proceso dirigido a evaluar la posición financiera, así como los resultados de las operaciones de una empresa. Los más usados son: el balance general y el estado de resultados. El surgimiento de la inteligencia artificial dio origen a sistemas que tienen la capacidad de realizar tareas complejas y con la capacidad de auxiliar a un usuario en un área específica, dando respuestas tal y como un experto lo haría. Las condiciones presentes en los mercados financieros dieron origen al desarrollo de herramientas que proporcionen información sobre la salud financiera de una empresa, necesitando modelos innovadores que logren dar solución a problemas que no pueden resolverse con métodos clásicos, sino con aquellos que proporciona la inteligencia artificial y la lógica difusa, ya que proporcionan los medios para apoyar la toma de decisiones de manera oportuna y precisa. En este artículo se propone el diseño de la arquitectura de un sistema experto basado en lógica difusa para el análisis de estados financieros XBRL de empresas mexicanas como apoyo en la toma de decisiones. La arquitectura se basa en un diseño de capas donde cada funcionalidad de los componentes se distribuye homogéneamente para facilitar la escalabilidad de la aplicación web.
\end{abstract}

Palabras clave: inteligencia artificial, lógica difusa, XBRL, análisis financiero, estados financieros, sistema experto, Web.

\section{Architecture for the Analysis of XBRL Financial Statements Published by Companies in México using Fuzzy Logic}

\begin{abstract}
The analysis of financial statements is the process aimed at evaluating the financial position, as well the results of the operations of a company, the most used are: The balance sheet and the income statement. The emergence of artificial
\end{abstract}




\begin{abstract}
intelligence gave rise to systems that have the ability to perform complex tasks and with the ability to assist a user in a specific area, giving answers as an expert would. The present conditions in the financial markets gave rise to the development of tools that provide information on the financial health of a company, needing innovative models that manage to solve problems that can not be solved with classical methods, if not, with those that provide intelligence, artificial and fuzzy logic, as they provide the means to support decision making in a timely and accurate manner. This article proposes the design of the architecture of an expert system based on fuzzy logic for the analysis of XBRL financial statements of Mexican companies as support in decision making. The architecture is based on a layer design where each functionality of the components is distributed homogeneously to facilitate the scalability of the web application.
\end{abstract}

Keywords: artificial intelligence, fuzzy logic, XBRL, financial analysis, financial statements, expert system, Web.

\title{
1. Introducción
}

El análisis de estados financieros es el proceso dirigido a evaluar la posición financiera, así como los resultados de las operaciones de una empresa, con el objetivo de establecer las mejores estimaciones y predicciones posibles sobre las condiciones y resultados futuros. El proceso de análisis de estados financieros consiste en la aplicación de herramientas y técnicas analíticas a los estados y datos financieros, con el fin de obtener de ellos medidas y relaciones que son significativas y útiles para la toma de decisiones.

Así, el análisis financiero cumple en primer lugar la función de convertir los datos en información útil [1]. Adicionalmente, durante el análisis de los estados financieros se identifican aspectos relevantes para el apoyo en la toma de decisiones. Desarrollar un análisis ayuda a evaluar el valor de los estados financieros de una empresa [2], complementando esta aseveración en [3] mencionan que el análisis fundamental tiene por objeto determinar el valor de los títulos privados mediante un examen cuidadoso de factores clave/valor, tales como los ingresos, las inversiones, el riesgo, el crecimiento y la posición competitiva, entre otros.

La lógica difusa está relacionada y fundamentada en la teoría de conjuntos difusos, según la cual el grado de pertenencia de un elemento a un conjunto está determinado por una función de pertenecia que puede tomar todos los valores reales comprendidos en el intervalo de $(0,1)[4]$.

Cuando la información es imprecisa o insuficiente, usar instrumentos estadísticos no basta para obtener resultados significativos. La lógica difusa surge precisamente para tratar con este tipo de problemas y lograr darles una solución óptima. De esta forma, una combinación entre un sistema de lógica difusa y la experiencia o conocimiento que tienen los encargados de tomar las decisiones es una excelente manera de obtener buenos resultados.

La aplicación de la lógica difusa permite enfrentar problemas de manera efectiva para la creación de sistemas de soporte para la toma de decisiones, ya que los modelos que se utilizan son altamente flexibles y más tolerantes a la imprecisión de los datos. Por otra parte, una razón financiera es un indicador esencial para conocer la situación 
Arquitectura para el análisis de estados financieros XBRL publicados por empresas en México ...

económica de una empresa [5], y se expresa por medio de una fórmula matemática específica y simple.

Las razones financieras proporcionan información que beneficia a los interesados en la toma de decisiones empresariales. Además, ayudan a determinar la magnitud y la dirección de los cambios en la empresa durante un período de tiempo. Actualmente, la estandarización se ha convertido en un problema importante para el desarrollo de varias industrias. Los estándares propician mejorar la calidad de los datos de la industria. En este sentido, XBRL (eXtensible Business Reporting Language), un lenguaje basado en XML (eXtensible Markup Language) que permite la publicación electrónica de datos comerciales y financieros, es aplicado a muchos dominios, incluida la supervisión financiera, regulación gubernamental y control interno de la corporación [6].

Es imperativo mencionar que en México a partir del primer trimestre de 2016 las emisoras industriales, comerciales y de servicios tienen la obligación de enviar su información financiera trimestral en formato XBRL [6]. Para finales de 2017 fueron publicadas nuevas taxonomías, por lo que se estimó que la Comisión Nacional Bancaria y de Valores (CNVB) publicara como fecha de inicio de la obligación para la publicación de reportes bajo este nuevo esquema a partir del 1 de enero de 2018 [7]. Como se puede obervar, la adopción e implementación del estándar XBRL en México esta en proceso de consolidación.

Considerando lo anterior, la principal contribución de este trabajo es proporcionar una arquitectura para un sistema experto basado en lógica difusa que realice el análisis de los estados financieros publicados en formato XBRL por empresas mexicanas, con el propósito de genenerar un sistema de análisis financiero que brinde soporte a la información publicada en formato XBRL, apoyado de la lógica difusa para la interpretación de resultados y como apoyo a la toma de decisiones financieras.

Este documento está estructurado de la siguiente manera: La Sección 2 presenta un conjunto de trabajos relacionados con esta propuesta, organizándolos en los temas que presentan el análisis financiero usando lógica difusa y el de análisis financiero con XBRL; en la Sección 3, se describe la arquitectura propuesta, las capas que la integran, los componentes de cada capa, así como el flujo de trabajo entre ellos; además, la sección 4 incluye un estudio de caso $\mathrm{y}$, finalmente, la sección 5 presenta las conclusiones y el trabajo futuro.

\section{Trabajos relacionados}

A continuación, se presenta una revisión del estado del arte en la que se toman en cuenta los aspectos del análisis financiero, la lógica difusa y el estándar XBRL.

\subsection{Análisis financiero y lógica difusa}

En [8] se desarrolló un sistema experto que soluciona problemas de autorización de crédito bancario. El sistema utiliza los resultados del análisis de crédito de los usuarios para crear una base de conocimientos cuyas entradas y parámetros se caracterizan por valores difusos, por esta razón el uso de un mecanismo de inferencia de lógica difusa se utilizó en el sistema para apoyar en la toma de decisiones para otorgar créditos bancarios. 
En [9] se proporcionó un sistema experto estadístico difuso para la gestión del flujo de efectivo. El sistema ayudó a administrar los recursos efectivos de la organización. Para ello, los autores definieron las variables de entrada, salida y sus funciones de pertenencia, se formaron reglas usando el sistema de inferencia difuso para inferir el saldo de efectivo final de un conjunto de combinación de 25 reglas separadas. Finalmente, los niveles lingüísticos se convirtieron a ciertos números por el método centrado para ayudar a ver los efectos de los cambios en los niveles de insumos en los saldos de efectivo finales.

En [10] se estudió y modeló un sistema de asignación de recursos financieros a compañías comisionistas de bolsa, con el fin de disminuir el riesgo de impago del capital asignado y, además; que estos capitales generen rendimientos adicionales para la empresa. El modelo planteado basado en sistemas expertos difusos permitió soportar estas decisiones de asignación de recursos financieros.

Arias-Aranda, et al. [11], diseñaron un sistema experto difuso centrado en aumentar la precisión y la calidad del conocimiento para la toma de decisiones. El sistema utilizó un modelo basado en reglas difusas para simular el comportamiento de las empresas, se presentó bajo el supuesto de parámetros de entrada determinados previamente detectados y se desarrolló un algoritmo para lograr la estructura mínima del modelo. El resultado obtenido fue una herramienta de sistema experto difuso, llamada ESROM, que proporciona información valiosa para ayudar a los gerentes a mejorar el logro de los objetivos de la empresa. Una de las principales aportaciones de la iniciativa presentada por los autores de este trabajo es que el sistema es general, y se adapta a diferentes escenarios.

En [12] se propuso la adopción de una técnica inteligente híbrida, un sistema experto difuso para llevar a cabo un análisis costo-beneficio de la inversión en sistemas de información empresarial. Los modelos tradicionales de presupuestación de capital se centran en variables cuantificables. Sin embargo, hay muchas variables intangibles que hacen que el uso de medidas completamente cuantitativas sea incompleto y menos inclusivo. Por lo que en este estudio se tomó gran conocimiento de las variables intangibles y de vaguedad en la toma de decisiones del grupo humano que requiere un alto nivel de consenso.

En los sistemas expertos existentes la incertidumbre se trata mediante una combinación de lógica predicativa y métodos basados en la probabilidad. Una deficiencia grave de estos métodos es que no son capaces de enfrentarse a la información difusa en la base de conocimientos. Por lo que un enfoque alternativo al manejo de la incertidumbre se sugierió en [13], el cual se basa en el uso de la lógica difusa.

En [14] se planteó un enfoque híbrido para la evaluación del desempeño financiero de las compañías automotrices de la bolsa de Teherán. Para ello, se estructuró un modelo jerárquico de evaluación del desempeño financiero basado en las medidas contables y las medidas de valor económico. En este enfoque se aplicó el Fuzzy Analytic Hierarchy Process para determinar los pesos de los criterios, también los resultados de tres métodos de superación se combinaron usando los rangos medios. Los resultados representaron que las medidas de valor económico son más importantes que las medidas contables en la evaluación del desempeño financiero de las empresas.

Los casos asociados con el análisis de riesgo del préstamo y las estrategias de adaptación relacionadas han crecido en importancia y complejidad, por lo que Kumar, 
Arquitectura para el análisis de estados financieros XBRL publicados por empresas en México ...

Bhatia y Kapoor [15] plantearon controlar este problema utilizando el conocimiento y la experiencia de expertos involucrados en este proceso y que tienen conocimiento sobre este campo. Por tal razón dichos autores proporcionaron el análisis de riesgo basado en un sistema de inferencia difusa, así como la interfaz gráfica de usuario que consideró los diferentes parámetros de un solicitante. El análisis de los resultados experimentales de los diferentes solicitantes comprobó la corrección y la coherencia de la decisión del sistema de apoyo para la toma de decisiones.

\subsection{Análisis financiero con $X B R L$}

El número de aplicaciones de software que permiten analizar informes XBRL es escaso, debido a la reciente estandarización de los informes financieros que utilizan XBRL, lo que la convierte en un área de estudio interesante y una fuente de oportunidades de investigación aprovechada por S. Mendez et al. [16], quienes agruparon estas aplicaciones en las dos categorías siguientes: 1) aplicaciones para la validación, edición y generación de informes contables, casi todas las aplicaciones de este tipo son privadas y tienen altos costos de licencia y, 2) un conjunto de herramientas que ayudan a los usuarios de los informes XBRL a evaluar la solvencia y la rentabilidad futura de las empresas en estudio. En [16] se propuso un enfoque para extraer información de conjuntos de documentos que cumplen con diferentes taxonomías XBRL en diferentes formatos. Esto se hace clasificando la información contable en una ontología de conceptos financieros diseñados para este propósito. El procesamiento automatizado de la información, que es posible debido al uso de XML, brinda a los usuarios la máxima flexibilidad en sus consultas.

En [17] se presentó una plataforma inteligente llamada FLORA, la cual provee un enfoque para hacer frente a las deficiencias actuales de información financiera y lograr una forma más efectiva de procesar datos financieros basados en los principios de Linked Data.

En éste se describió el proceso de extracción de datos y el modelado semántico que son los pilares del análisis de datos financieros. Como resultado, FLORA facilita un análisis financiero eficaz, basado en datos, y una integración a escala web entre las aplicaciones financieras y las plataformas.

\subsection{Análisis comparativo de los trabajos relacionados}

A continuación, se presenta un breve análisis comparativo entre los trabajos relacionados y nuestra iniciativa. La Tabla 1 indica si el trabajo presentado describe su arquitectura, el tipo de estados financieros que analiza, el tipo de análisis que se lleva a cabo sobre la información de origen, y si ofrecen soporte al estándar XBRL.

Como se observa en la Tabla 1, el desarrollo e investigación de sistemas expertos para el análisis financiero, utilizando lógica difusa, es cada vez más extendido y ocurre en diferentes contextos como en [8, 9, 10 y 15] que se enfocan en el análisis de riesgos para la autorización de créditos y préstamos, la asignación de recursos; sin embargo, es importante destacar que pocas iniciativas de las analizadas en la literatura presentan una arquitectura que permita la integración de lógica difusa como parte esencial del análisis de estados financieros publicados bajo el estándar XBRL como en [16] y [17]; 
Cristian Noé Enríquez Marcial, Hilarion Miñoz Contreras, José Luis Sánchez Cervantes, et al.

Tabla 1. Análisis comparativo entre los trabajos relacionados y el presente trabajo.

\begin{tabular}{ccccc}
\hline Iniciativa & Arquitectura & $\begin{array}{c}\text { Contexto del análisis } \\
\text { financiero }\end{array}$ & $\begin{array}{c}\text { Tipo de } \\
\text { análisis }\end{array}$ & $\begin{array}{c}\text { Soporte } \\
\text { XBRL }\end{array}$ \\
\hline $\begin{array}{c}\text { M. Menekay. } \\
\text { [8] }\end{array}$ & No & $\begin{array}{c}\text { Autorización de créditos } \\
\text { bancarios }\end{array}$ & $\begin{array}{c}\text { Sistema de } \\
\text { inferencia } \\
\text { difuso }\end{array}$ & No \\
\hline $\begin{array}{c}\text { A. Anvary } \\
\text { Rostamy et. } \\
\text { al. [9] }\end{array}$ & No & Flujo de efectivo & $\begin{array}{c}\text { Sistema de } \\
\text { inferencia } \\
\text { difuso }\end{array}$ & No \\
\hline $\begin{array}{c}\text { S. Medina } \\
\text { Hurtado et. al. } \\
\text { [10] }\end{array}$ & No & $\begin{array}{c}\text { Asignación de recursos } \\
\text { financieros a compañías } \\
\text { comisionistas }\end{array}$ & $\begin{array}{c}\text { Sistema de } \\
\text { inferencia } \\
\text { difuso }\end{array}$ & No \\
\hline $\begin{array}{c}\text { S. Kumar et. } \\
\text { al. [15] }\end{array}$ & No & $\begin{array}{c}\text { Análisis de riesgo del } \\
\text { préstamo }\end{array}$ & $\begin{array}{c}\text { Sistema de } \\
\text { inferencia } \\
\text { difuso }\end{array}$ & No \\
\hline $\begin{array}{c}\text { S. Mendez } \\
\text { Nuñez et. al. } \\
{[16]}\end{array}$ & Sí & $\begin{array}{c}\text { Balance general } \\
\text { Estado de resultados }\end{array}$ & $\begin{array}{c}\text { Tecnologías de } \\
\text { Web Semántica }\end{array}$ & Sí \\
\hline $\begin{array}{c}\text { M. Radzimski } \\
\text { et. al. [17] }\end{array}$ & Sí & $\begin{array}{c}\text { Balance general } \\
\text { Estado de resultados } \\
\text { Flujo de efectivo }\end{array}$ & $\begin{array}{c}\text { Tecnologías de } \\
\text { Web Semántica }\end{array}$ & Sí \\
\hline $\begin{array}{c}\text { Nuestra } \\
\text { iniciativa }\end{array}$ & Sí & $\begin{array}{c}\text { Balance general } \\
\text { Estado de resultados } \\
\text { Flujo de efectivo }\end{array}$ & $\begin{array}{c}\text { Sistema de } \\
\text { inferencia } \\
\text { difuso }\end{array}$ & Sí \\
\hline
\end{tabular}

no obstante, estas iniciativas no analizan estados financieros publicados en México y no incluyen lógica difusa para la generación de recomendaciones financieras.

Adicionalmente, nuestra iniciativa incluye en el diseño una arquitectura organizada en capas para su fácil mantenimiento, misma que se es descrita brevemente en la siguiente sección.

\section{Arquitectura}

La arquitectura que se muestra en la Fig. 1, se basa en cuatro capas. Cada capa contiene componentes, algunos de ellos se conforman por subcomponentes. Las tareas y responsabilidades de la aplicación se distribuyen entre sus distintos componentes. La arquitectura diseñada permite fácil escalabilidad y mantenimiento.

Asimismo, la arquitectura mostrada en la Fig. 1, ayuda a comprender gráficamente los componentes estructurales de los cuales se integra las relaciones entre ellos, así como el flujo de trabajo que se llevará a cabo para la comunicación y la obtención de resultados entre componentes.

\subsection{Descripción de capas}

Cada una de las capas tiene una función explicada a continuación: 


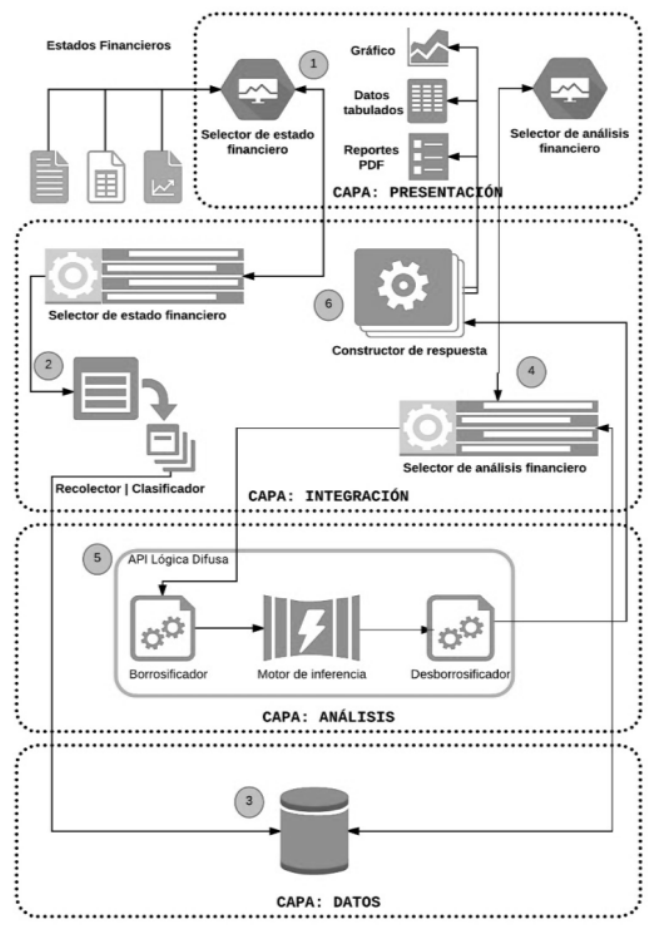

Fig. 1. Arquitectura del sistema experto difuso.

- Capa de presentación: Esta capa representa la interfaz entre el usuario y la aplicación. Contiene los componentes que hacen posible la interacción del usuario con la aplicación.

- Capa de integración: Esta capa contiene los componentes necesarios para enviar y recibir los datos e interactuar con la capa de presentación. Esta capa incluye el selector de estado financiero, así como la construcción de la información que será mostrada al usuario.

- Capa de análisis: Esta capa contiene la Interfaz de Programación de Aplicaciones (API) de lógica difusa, que será la encargada de borrosificar los datos para posteriormente realizar el análisis financiero y entregará un resultado, el cual será entregado al constructor de respuesta.

- Capa de datos: Para esta arquitectura ha sido considerado tener un repositorio de información para almacenar los datos que han sido extraídos y clasificados para su posterior análisis, así como almacenar los resultados de los análisis realizados.

\subsection{Descripción de componentes}

Los componentes que forman parte de cada capa de la arquitectura (Fig. 1), tienen funciones que definen su comportamiento, el cual se describe brevemente a continuación: 
- Selector de estado financiero: Proporciona una Interfaz Gráfica de Usuario (GUI) desarrollada a través del marco de trabajo Spring MVC (Modelo Vista Controlador) y componentes de PrimeFaces, este último para el diseño responsivo de la página para que se adapte a la pantalla del dispositivo que la visualiza. A través de este componente, el usuario tiene la opción de elegir el estado financiero que será objeto de análisis por el sistema, la GUI proporciona la interfaz para que el usuario provea la información de los estados financieros en formato XBRL, Valores separados por Comas (CSV), y la Notación de objetos de JavaScript (JSON), entre otros.

- Recolector: Es el encargado de recuperar y limpiar la información enviada por el usuario mediante el componente selector de estado financiero.

- Clasificador: Una vez hecha la limpieza de la información por el módulo de recolección, este componente se encargará de realizar la clasificación de los datos para almacenarlos en el componente base de datos.

- Selector de análisis financiero: A través de este componente, el usuario tiene la opción de elegir el tipo de análisis financiero que será realizado por el sistema a la información obtenida de la base de datos, la cual será proporcionada al componente de la API de lógica difusa.

- Constructor de respuesta: Este componente recibe las respuestas de la API de lógica difusa después de la ejecución del análisis seleccionado, recupera la información y crea un reporte de análisis financiero, dicho documento se presentará en formato gráfico visual, en un reporte PDF o en datos tabulados para apoyar al usuario en la toma de decisiones.

- API Lógica difusa: Este componente recibe la información recuperada por el selector de análisis financiero, para realizar su procesamiento. Su función principal es realizar la borrosificación de los datos recuperados para después, realizar el análisis mediante el motor de inferencia donde previamente se han definido las reglas y conjuntos de pertenencia de la información, una vez analizada dicha información se procede a la desborrosificar los datos para obtener las razones financieras de análisis y generar una respuesta mediante el constructor de respuesta.

- Base de datos: La función de este componente es almacenar la información que mediante un proceso de extracción, limpieza y transformación es recuperada por el módulo recolector/clasificador de la aplicación. De la misma manera que almacena los resultaos generados por el selector de análisis financiero.

\subsection{Flujo de trabajo}

Las relaciones entre los componentes de la arquitectura que se muestran en la Fig. 1 definen el flujo de trabajo para el proceso del análisis de estados financieros, desde la selección del tipo de dato de entrada, hasta la elección de como el usuario desea se presenten los resultados. Aquí hay una breve descripción del flujo de trabajo de la arquitectura:

1. A través de la GUI desarrollada usando el marco de trabajo Spring MVC, el usuario realiza una solicitud basada en el protocolo HTTP al ingresar mediante un selector de archivo situado en la capa de presentación, el estado financiero a analizar. El componente selector de estado financiero situado en la capa de integración envía el documento proporcionado por el usuario a componente recolector/clasificador. 
Arquitectura para el análisis de estados financieros XBRL publicados por empresas en México ...

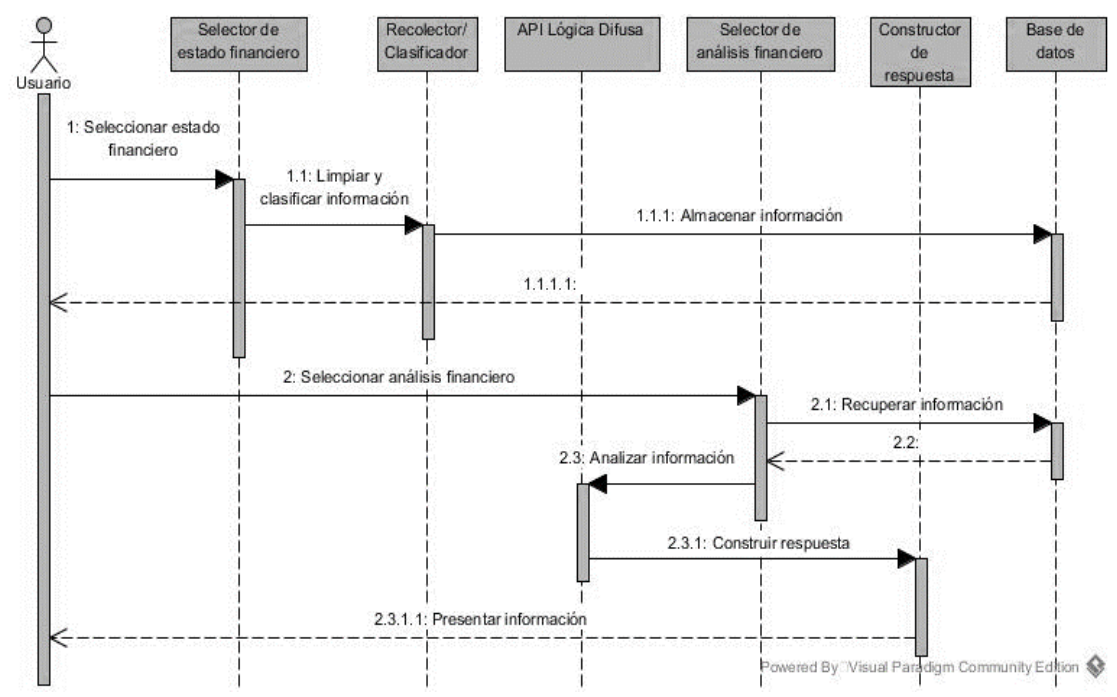

Fig. 2. Diagrama de secuencia del flujo de trabajo de la arquitectura.

2. La información será extraída del documento por el componente recolector/clasificador para realizar la limpieza y clasificación de los datos.

3. Los datos extraídos por el componente recolector/clasificador será almacenada en la base de datos.

4. Por medio del componente selector de análisis financiero situado en la capa de presentación, el usuario seleccionará el tipo de análisis que se aplicará a la información almacenada en la base de datos. El componente selector de análisis financiero situado en la capa de integración enviará el tipo de análisis a realizar y la información a la API de lógica difusa.

5. La API de lógica difusa hará la borrosificacion de la información proporcionada, mediante el motor de inferencia, el cual contiene las reglas proporcionadas por un experto, será el encargado de realizar el análisis financiero y definir el conjunto de pertenencia de la información, la cual será desborrosificado para su interpretación.

6. La respuesta proporcionada por la API de lógica difusa será enviada al componente constructor de respuesta situado en la capa de integración que será el encargado de presentar la información de forma visual para el usuario y así apoyar en la toma de decisiones.

En la Fig. 2, se muestra un diagrama de secuencia basado en el Lenguaje Unificado de Modelado (UML) con una descripción detallada el flujo de trabajo descrito previamente.

\section{Estudio de caso}

Para este caso se parte del supuesto de que una persona desea invertir capital en una empresa, por lo que es necesario realizar un análisis financiero. El análisis financiero 


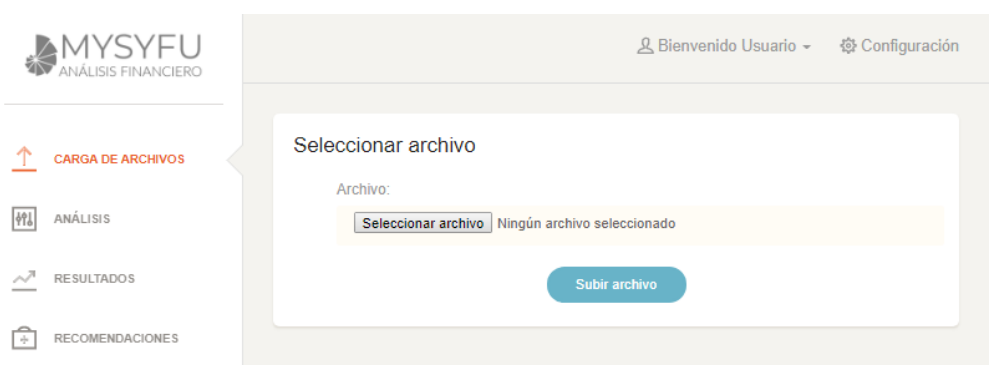

Fig. 3. Interfaz de carga de documentos XBRL.
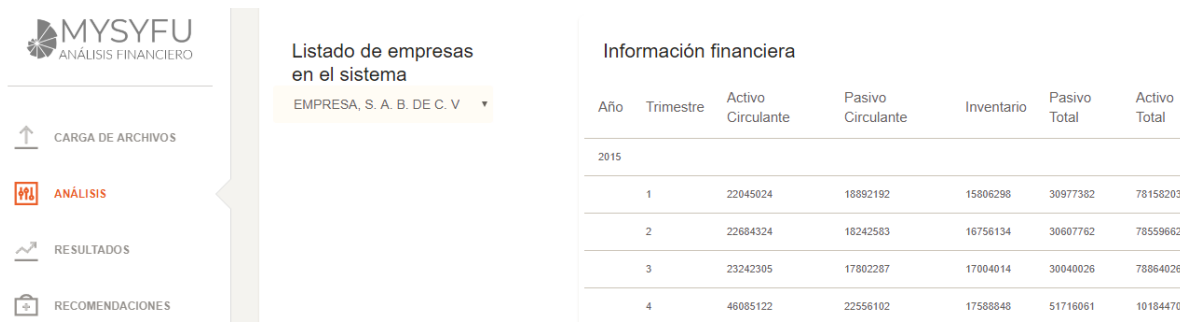

Fig. 4. Información financiera extraida de los estados financieros XBRL.

Tabla 1. Fórmulas para calcular algunas razones financieras.

\begin{tabular}{cc}
\hline Ratio Financiero & Fórmula \\
\hline Razón corriente & Razón corriente $=\frac{\text { Activo corriente }}{\text { Pasivo Circulante }}$ \\
\hline Capital de trabajo & Capital de trabajo $=\frac{(\text { Activo circulante }- \text { Pasivo Circulante })}{\text { Pasivo Circulante }}$ \\
\hline Prueba acida & Prueba Ácida $=\frac{(\text { Activo circulante }- \text { Inventarios })}{\text { Pasivos Circulantes }}$ \\
\hline Razón de deuda & Razón de deuda $=\left(\frac{\text { Pasivos totales }}{\text { Activos totales }}\right) * 100$ \\
\hline
\end{tabular}

busca obtener una recomendación para invertir a partir del cálculo automático de los siguientes indicadores financieros:

A) Razón corriente: Permite determinar el índice de liquidez de una empresa;

B) Capital de trabajo: Busca garantizar las operaciones de la empresa, si el resultado es positivo, da la posibilidad de generar inversión y si es negativo, da la posibilidad de buscar financiamiento propio o mediante fondos de terceros;

C) Prueba Ácida: Revela la capacidad de la empresa para cancelar sus obligaciones corrientes, pero sin contar con la venta de sus existencias y, 
Arquitectura para el análisis de estados financieros XBRL publicados por empresas en México ...

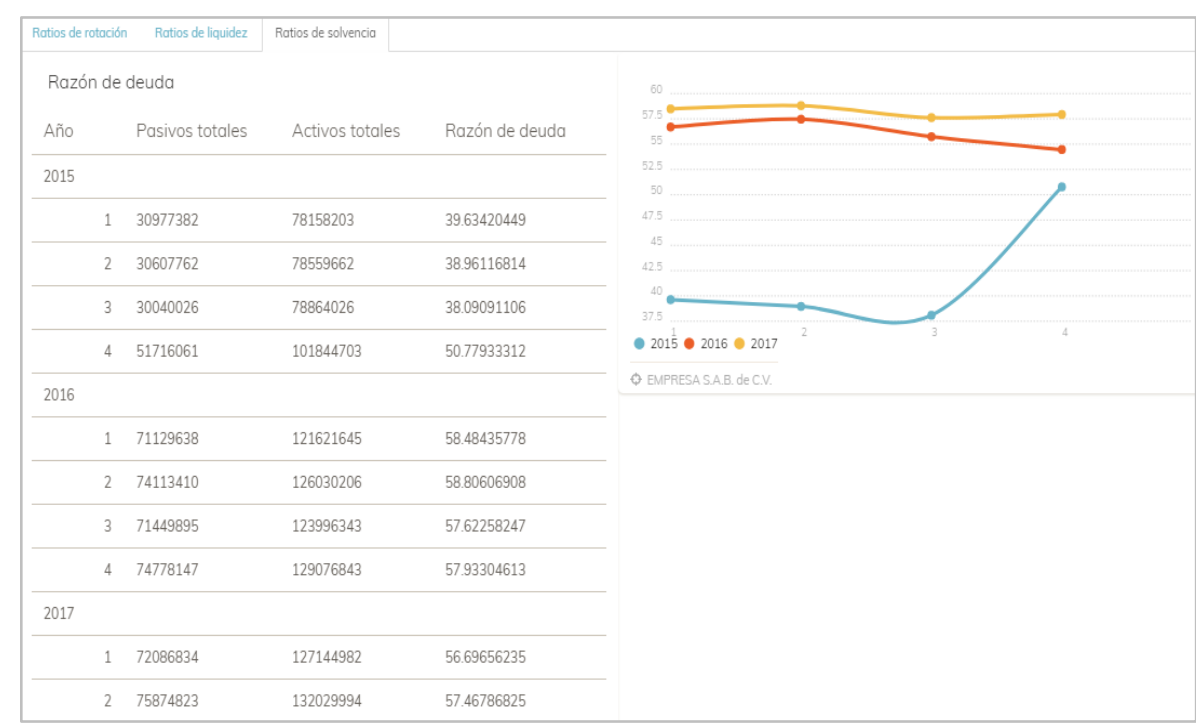

Fig. 5. Información del cálculo de las razones financieras.

Tabla 3. Indicador de Razón corriente por año.

\begin{tabular}{cccccc}
\hline Año & $\begin{array}{c}\text { Activo } \\
\text { Circulante }\end{array}$ & $\begin{array}{c}\text { Pasivo } \\
\text { Circulante }\end{array}$ & $\begin{array}{c}\text { Razón } \\
\text { Corriente }\end{array}$ & Obligaciones & Disponible \\
\hline $\mathbf{2 0 1 5}$ & 114056775 & 77493164 & 1.471830147 & 67.94262244 & 32.05737756 \\
\hline $\mathbf{2 0 1 6}$ & 136758411 & 127231863 & 1.074875489 & 93.03403138 & 6.965968623 \\
\hline $\mathbf{2 0 1 7}$ & 151602198 & 146930010 & 1.031798732 & 96.91812648 & 3.081873523 \\
\hline
\end{tabular}

Tabla 4. Indicador de Prueba ACID.

\begin{tabular}{ccccc}
\hline Año & Activo Circulante & Pasivo Circulante & Inventario & $\begin{array}{c}\text { Prueba } \\
\text { Acida }\end{array}$ \\
\hline $\mathbf{2 0 1 5}$ & 114056775 & 77493164 & 67155294 & 0.605233786 \\
\hline $\mathbf{2 0 1 6}$ & 136758411 & 127231863 & 96461883 & 0.316717268 \\
\hline $\mathbf{2 0 1 7}$ & 151602198 & 146930010 & 110481272 & 0.279867442 \\
\hline
\end{tabular}

Tabla 5. Indicador de Razón de deuda.

\begin{tabular}{lccc}
\hline Año & Pasivos totales & Activos totales & Razon de deuda \\
\hline $\mathbf{2 0 1 5}$ & 143341231 & 337426594 & 42.48071538 \\
\hline $\mathbf{2 0 1 6}$ & 291471090 & 500725037 & 58.20980947 \\
\hline $\mathbf{2 0 1 7}$ & 290419217 & 517692020 & 56.09883981 \\
\hline
\end{tabular}

D) Razón de deuda: permite establecer el grado de participación de los acreedores, en los activos de la empresa. Mide la proporción de la inversión de la empresa que ha 
sido financiada por deuda, por lo que se acostumbra a presentar su resultado en forma de porcentaje.

Tales indicadores permiten agilizar el diagnóstico de la situación actual de la empresa y facilita la toma de su decisión para realizar una inversión. Con la solución propuesta, se pretende que la persona interesada pueda obtener una recomendación para realizar la toma de decisiones financieras, por lo que lleva a cabo el proceso siguiente:

A partir de un conjunto de datos proporcionados en formato XBRL se obtiene la información que permite realizar el análisis de los estados financieros de la empresa, como lo son el estado de situación financiera, el estado de resultados, el estado de flujo de efectivo, entre otros. En la Fig. 3, se muestra la interfaz para la carga de los estados financieros de la empresa a analizar.

De los datos extraídos se obtiene la información financiera del periodo comprendido entre los años 2015 y 2017 para la empresa, a partir de esta se calcularán las razones financieras aplicando las formulas que se muestra en la Tabla 2, una vez realizado el calculo de las razones, la información se visualiza como se muestra en la Fig. 4.

Las razones financieras son unos indicadores que profundizan en la información de los estados financieros, estos están catalogados en ratios de liquidez, solvencia, rendimiento y apalancamiento. En la Fig. 5, se presenta la información obtenida del cálculo de las razones.

A partir del valor obtenido para el cálculo de la razón corriente que se muestra en la Tabla 3 en el año 2015 se da la siguiente interpretación: por cada peso de obligación vigente (deuda), la empresa contaba con 1.47 pesos para respaldarla.

Con los valores obtenidos para la prueba acida, los cuales observamos en la Tabla 4, se obtuvo que en los años más recientes 2016 y 2017, la empresa mantuvo valores de entre 0.31 y 0.22 respectivamente, los cuales, están por debajo del valor óptimo indicado para esta prueba (0.50 a 1.0), lo que significa que la empresa, si en algún momento tuvo la necesidad de atender todas sus obligaciones corrientes sin la necesidad de liquidar y vender sus inventarios, a esta, no le habría alcanzado y, por lo tanto, habría tenido que vender sus inventarios (o parte de ellos) para poder cumplir con sus obligaciones.

Aplicando la formula dada anteriormente, se calcula la Razón de deuda de la empresa, de esta manera, analizan e interpretan los resultados obtenidos y mostrados por año en la Tabla 5., los cuales indican que para el año 2015, el $42 \%$ del total de la inversión (Activos Totales) fue financiado con la participación de sus acreedores.

Esta situación aumento para el 2016 por lo que representa un moderado nivel de riesgo inversión para la empresa.

El caso de estudio presentado muestra la realización del análisis de estados financieros a partir de su representación en formato XBRL, por lo que se continuará con el desarrollo de un módulo de lógica difusa que analizará los indicadores financieros de manera automática con el propósito de generar recomendaciones que permitan apoyar en la toma de decisiones al usuario final.

\section{Conclusiones y trabajo futuro}

Los aspectos tratados en el presente trabajo servirán como base para el desarrollo de una herramienta de autoría. Se presentó una arquitectura que, mediante el uso de 
Arquitectura para el análisis de estados financieros XBRL publicados por empresas en México ...

tecnologías Web y lógica difusa facilitará el análisis de estados financieros publicados por las empresas mexicanas utilizando el estándar XBRL. Los datos contenidos en los informes XBRL son un gran avance para la estructuración y el análisis de la información financiera. La implementación de la arquitectura propuesta aumentaría la eficiencia de los procesos de toma de decisiones a través de la incorporación de un módulo de lógica difusa que permita generar recomendaciones con la finalidad de apoyar en la toma de decisiones financieras empresariales.

Como trabajo a futuro se implementarán dos modulos, el primero será el de lógica difusa que genere las recomendaciones automáticas necesarias para dar soporte a la toma de decisiones utilizando inferencia basada en reglas apoyado en la arquitectura propuesta, el segundo módulo permitirá exportar los resultados obtenidos en los formatos comunes incluyendo hojas de cálculo, PDF, JSON y XML.

Finalmente, se considera el uso de técnicas de aprendizaje profundo que permitan la generación de analítica predictiva para detectar interacciones en los datos financieros nuevos e históricos que pueden pasar desapercibidas, pero que ayudarán a obtener el conocimiento necesario para la creación de modelos predictivos que de manera automática, apoyen en la toma de decisiones para predecir el comportamiento y tendencias de las empresas con base en la información expuesta en sus estados financieros.

Agradecimientos. Los autores de este documento agradecen el apoyo del Consejo Nacional de Ciencia y Tecnología (CONACYT) así como al Tecnológico Nacional de México (TecNM) por el apoyo para la realización de este trabajo.

\section{Referencias}

1. Bernstein, L.A.: Análisis de estados financieros: teoría, aplicación e interpretación, 657.31/B53fE (1995)

2. Ou, J.A., Penman, S.H.: Financial statement analysis and the prediction of stock returns. J. Account. Econ., 11(4), pp. 295-329 (1989)

3. Lev, B., Thiagarajan, S.R.: Fundamental information analysis. J. Account. Res., pp. 190215 (1993)

4. Kulkarni, A. D.: Computer vision and fuzzy-neural systems. Prentice Hall PTR (2001)

5. Bliss, J.H.: Financial and operating ratios in management. The Ronald press company (1923)

6. Santos-Morales, R.A.R.J.N., Muguerza-Medina L.: Bolsa Mexicana de Valores (2016) https://www.bmv.com.mx/work/models/Grupo_BMV/Resource/1928/Presentacion_XBRL _Portal_10feb16_7.56.pdf (2018)

7. 2H Software: XBRL México. http://www.xbrl.mx/Noticias2HTaxonomia.aspx (2018)

8. Menekay, M.: Bank Credit Authorization Using Fuzzy Expert System. Procedia Comput. Sci., 102, pp. 659-662 (2016)

9. Rostamy, A.A., Baghaei,V.F., Takanlou, B., Rostamy, A.A.: A fuzzy statistical expert system for cash flow analysis and management under uncertainty. Adv. Econ. Bus., 1(2), pp. 89-102 (2013)

10. Medina-Hurtado, S., Manco, O.O.: Design of a fuzzy expert system: Credit risk assessment of stock brokerage firms in granting financial resources. Estud. gerenciales, 23(104), pp. 101-129 (2007)

11. Arias-Aranda, D., Castro, J.L., Navarro, M., Sánchez,. J.M., Zurita, J.M.: A fuzzy expert system for business management. Expert Syst. Appl., 37(12), pp. 7570-7580 (2010) 
Cristian Noé Enríquez Marcial, Hilarion Miñoz Contreras, José Luis Sánchez Cervantes, et al.

12. UzokaF, E.: Fuzzy-expert system for cost benefit analysis of enterprise information systems: a framework. Int. J. Comput. Sci. Eng., 1(3), pp. 254-262 (2009)

13. Zadeh, L.A.: The role of fuzzy logic in the management of uncertainty in expert systems. Fuzzy sets Syst., 11(1-3), pp. 199-227 (1983)

14. Safaei-Ghadikolaei, A., Khalili Esbouei, S., Antucheviciene, J.: Applying fuzzy MCDM for financial performance evaluation of Iranian companies. Technol. Econ. Dev. Econ., 20(2), pp. 274-291 (2014)

15. Kumar, S., Bhatia, N., Kapoor, N.: Fuzzy logic based decision support system for loan risk assessment. In: Proceedings of the International Conference on Advances in Computing and Artificial Intelligence, pp. 179-182 (2011)

16. Núñez, S.M., Andrés-Suárez, J., Gayo, J.E.L., De Pablos, P.O.: A semantic based collaborative system for the interoperability of XBRL accounting information. In: World Summit on Knowledge Society, pp. 593-599 (2008)

17. Radzimski, M., Sanchez-Cervantes, J.L., Garcia-Crespo, A., Temiño-Aguirre. I.: Intelligent architecture for comparative analysis of public companies using semantics and XBRL data. Int. J. Softw. Eng. Knowl. Eng., 24(5), pp. 801-823 (2014) 